ITC 2/50

Information Technology and Control

Vol. 50 / No. 2/ 2021

pp. 390-405

DOI 10.5755/j01.itc.50.2.28210
Whale Optimization Algorithm with Applications to Power Allocation in Interference Networks

Received 2020/11/26

Accepted after revision 2021/02/02

HOW TO CITE: Du, Y., Zhang, X., Zhang, W., Wang, Z. (2021). Whale Optimization Algorithm with Applications to Power Allocation in Interference Networks. Information Technology and Control, 50(2), 390-405. https://doi.org/10.5755/j01.itc.50.2.28210

\title{
Whale Optimization Algorithm with Applications to Power Allocation in Interference Networks
}

\section{Yongwen Du, Xiquan Zhang, Wenxian Zhang and Zhangmin Wang}

School of Electronics and Information Engineering, Lanzhou Jiaotong University, Lanzhou 730070, China.

Corresponding author:2619036@stu.lzjtu.edu.cn

Power allocation plays a pivotal role in improving the communication performance of interference-limited wireless network (IWN). However, the optimization of power allocation is usually formulated as a mixed-integer non-linear programming (MINLP) problem, which is hard to solve. Whale optimization algorithm (WOA) has recently gained the attention of the researcher as an efficient method to solve a variety of optimization problems. WOA algorithm also has the disadvantages of low convergence accuracy and easy to fall into local optimum. To solve the above problems, we propose Cosine Compound Whale Optimization Algorithm (CCWOA). First of all, its unique cosine nonlinear convergence factor can balance the rate of the whole optimization process and prevent the convergence speed from being too fast. Secondly, the inertia weight and sine vector can increase the probability of jumping out of the local optimal solution. Finally, the Archimedean spiral can reduce the risk of losing the optimal solution. A representative benchmark function is selected to test the convergence rate of CCWOA algorithm and the optimization performance of jumping out of local optimum. Compared with the representative algorithms PFP and GAP, the optimization effect of CCWOA is almost consistent with the above two algorithms, and even exceeds $4 \%-6 \%$ in numerical value. The advantage of CCWOA is that it has lower algorithm complexity, which has a good advantage when the network computing resources are fixed. In addition, the optimization effect of CCWOA is higher than that of WOA, which lays a good foundation for further application of swarm intelligence optimization algorithm in network resource allocation.

KEYWORDS: Interference network, Power allocation, WOA, Archimedes spiral, Nonlinear convergence factor. 


\section{Introduction}

The wireless networks have been widely used in civilian and military fields, and people rely on wireless networks to transmit crucial private information. Due to broadcasting nature of wireless channel, how to ensure the security of communication with eavesdroppers become a great challenge [13, 21]. Security is a crucial issue in constructing and maintaining wireless communication networks. Shannon's paper [25] describes an unusual case now called an eavesdropping channel. In this case, the legitimate senders want to communicate securely with the legitimate receivers in the eaves-dropper's presence. Senders hope to transmit confidential information to receivers. However, it is difficult to rule out the influence of eavesdroppers on communication. Eavesdroppers may illegally extract information from an ongoing transmission without being detected $[3,18]$.

The physical layer security is becoming an emerging research direction. It is a way to use the transmission channel's inherent randomness to ensure safety, which is more reliable than traditional key-based encryption algorithms [28]. Wyner first mentions the concept of physical layer security [33]. Scholar studies the security of single input and single out-put eavesdropping channels and analyzes the physical layer security issues [29]. Scholar aims to optimize the secrecy throughput of wireless network with the presence of eavesdroppers, which is the difference between user throughput and eavesdropper throughput. Optimizing security energy efficiency (SEE) is also an important research goal indicated by the ratio of secrecy throughput to total network power consumption [22]. Thus far, the security researches of physical layer are mainly divided into two directions: optimizing secrecy throughput and improving security energy efficiency. Follow up research on physical layer security are based on it. Using ground terminals to transmit artificial noise can improve the confidential throughput of system by influencing the capacity of eavesdropping channel [9, 15]. In [9, 35], to increase the channel throughput of legal channel, coordination and cooperation is employed in the broadcast channel. In [31, 38], scholars study beamforming technology, which by designing the direction of transmitted signal, signal received by the legitimate receiver is enhanced. It not only improves the throughput of legal channel, but also reduces the throughput of eavesdropping channel. In [26], a path-following procedures (PFP) of low complexity and rapid convergence is developed to achieve optimal power allocation in multi-user interference networks. [29] and [30] describe power control algorithms that maximize SEE in decode forward and amplify forward relay networks, assuming that channel state information (CSI) is available. In [12], scholars propose a distributed power control algorithm for maximizing SEE direction finding relay protection. In [32], a general algorithm procedure (GAP) embedded with an iterative power allocation algorithm (IPAA) is proposed, which solves the basic trade-off between energy efficiency (EE) and spectral efficiency (SE).

Intelligent optimization algorithm is a kind of meta-heuristic algorithm. Because of its simple structure and low computational complexity, it has been widely used to optimize resources in wireless networks, such as power allocation for spectrum and energy efficiency. In [7], MOMGWO was proposed to solve the multi-objective optimization problem in the spectrum sensing field of cognitive radio networks. In [37], a new Ant Colony System is proposed for computing the node-disjoint optimal transmission energy consumption route for coded cooperative mobile networks. The author uses the WOA to control the parameters (weights and biases) in the training feedforward neural network [2].

In the communication network, how to ensure the secure communication between the two parties has always been a research hotpot. In this paper, we use an interference-restricted information transfer model to ensure the communication quality of legitimate users. The task of this paper is to optimize the minimum secure throughput (MST) of eavesdropper and SEE of transmission link by using CCWOA to control power allocation.

WOA is an intelligent optimization algorithm proposed by Mirjalili, which imitates the hunting behavior of humpback whales [19]. WOA has been widely used in various fields because of its unique optimization mechanism [14]. However, WOA and GWO [20], RFO [23], MPA [36] intelligent optimization algorithms have similar shortcomings, such as premature convergence and easy to fall into local optimization [5]. To 
overcome them, [36] introduce some chaotic variables, which combine the parameters of WOA with the chaotic map, effectively improves the convergence accuracy. To improve the local search ability, a hybrid algorithm combining tabu search and WOA is proposed in [1]. [10] uses reverse learning for initialization and offers an opposition -based whale optimization algorithm to enhance the search space's algorithm exploration. The above improvements only improve the performance of a single aspect, and few people study the overall optimization. This provides a new direction for improving the overall performance of WOA in the future.

We analyze the search mechanism of WOA and find that its search mechanism has defects. With the increase of the number of iterations, the linear convergence factor of WOA will lead to premature convergence and easy to fall into local optimum. Therefore, when WOA is used in large-scale mixed nonlinear programming problems, such as network resources, it is not suitable to solve multiple optimization values. Based on the improvement direction, CCWOA proposes two improvement strategies.

1 The contribution lies in the use of a new type of convergence factor-cosine vector, convergence rate problem in the iterative process of nonlinear equilibrium. We are also inspired by the particle swarm optimization (PSO), the idea of inertia weight is introduced into this algorithm, which can adjust the best position of whale in time.

2 Then, replace the logarithmic spiral in the original WOA with an equidistant Archimedean spiral. The equidistant spiral can reduce the risk of losing the optimum whale position due to the iteration step too large of the logarithmic spiral. On the one hand, the new sine vector will increase the randomness of selection and enhance the probability of finding the global optimum.

\section{System Model and Problem Description}

\subsection{System Model}

As shown in Figure 1, an IWN is composed of M single antenna transmitters and M users. Each group of communication links consists of a transmitter and a user. Single-antenna transmitter hopes to transmit confidential information to user secretly. Confidential information is denoted as $S_{i}$. It is required that eavesdropper can get information as few as possible when the IWN is working. Secrecy throughput is used as performance indicator. It includes optimizing the MST of the eavesdropper and the SEE of legitimate receivers.

Denote by $P_{i}$ the transmit power of transmitter $i$ and $P=\left(P_{1}, \cdots, P_{M}\right)^{T}$. Assume that $h_{j i}$ represents the channel gain from transmitter $j$ to user $i$. The received signal at user $i$ is

$$
y_{i}=h_{i i} P_{i} S_{i}+\sum_{j \neq i}^{M} h_{j i} P_{j} S_{j}+n_{i},
$$

where $n_{i} \in \mathcal{C N}\left(0, \sigma_{i}^{2}\right)$ is additive noise.

\section{Figure 1}

Interference network user model

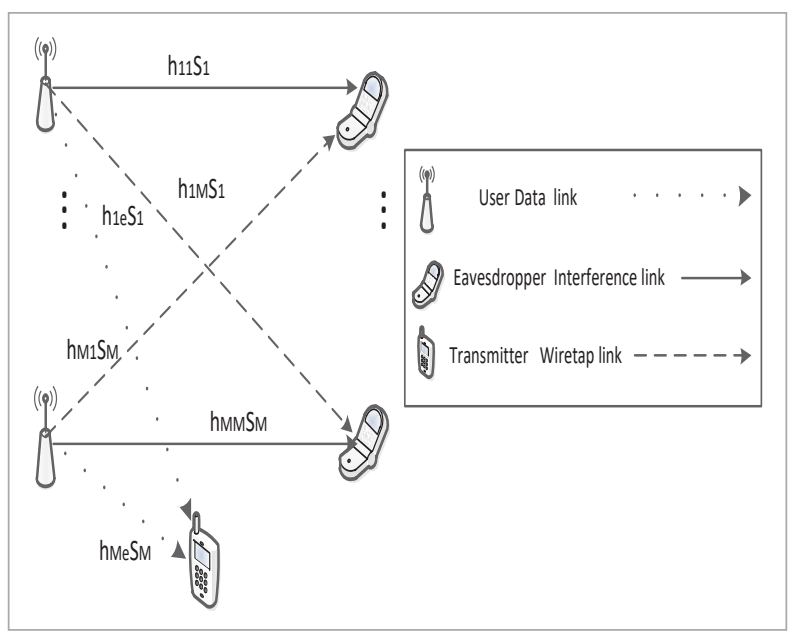

Suppose that an eavesdropper exists in the communication link, which is also receives signal from transmitters. Denote by $h_{i e}$ channel gain from transmitters $i$ to eavesdropper, receive signal at the eavesdropper is

$Y_{e}=\sum_{i=1}^{M} h_{i e} P_{i} S_{i}+n_{e}$,

where $n_{e} \in \mathcal{C N}\left(0, \sigma_{i}^{2}\right)$ is additive noise.

Under the perfect CSI of the transmitter, the information throughput of user $i$ is

$$
f_{i}(p) \triangleq \ln \left(1+\frac{h_{i i} P_{i}}{\sum_{j \neq i}^{M} h_{j i} P_{j}+\sigma_{i}^{2}}\right) .
$$


When eavesdropping channel is regarded as part of the communication link, assuming its channel gain $h_{i e}$ is known [32]. The eavesdropping throughput of eavesdropper user $i$ is

$$
g i(p) \triangleq \ln \left(1+\frac{h_{i e} P_{i}}{h_{j e} P_{j}+\sigma_{e}^{2}}\right) .
$$

When the transmitter sends information $S_{i}$ to user $i$, the secrecy throughput for eavesdropping are defined as $\max \left\{f_{i}(p)-g_{i}(p), 0\right\}$. Concurrently, energy efficiency is the ratio of throughput total network power consumption. The trade-off between EE and spectral SE can maximize SEE under the constraints of minimum rate requirements and transmit power budget. The total link power consumption $P_{\text {tot }}$ mainly has two parts: transmission power consumption $P_{i}$ and circuit power consumption $P_{i}^{C}$, so the total power consumption is defined as

$$
P_{t o t}(p)=\sum_{i=1}^{M}\left(\xi_{i} p_{i}+p_{i}^{c}\right) .
$$

In a network, we consider optimizing the following two fundamental issues.

\subsection{Problem Analysis}

1 The MST problem can be formulated as follows

$$
\begin{aligned}
& \max _{p} \Phi(p)=\min _{i=1, \ldots, M}\left[R_{i}(p)-\Gamma_{i}(p)\right] \\
& \text { s.t. } 0 \leq p_{i} \leq p_{i}^{\max }, \forall i=1, \ldots, M,
\end{aligned}
$$

where $P_{i}^{\max }$ represents the peak transmit power of user $i$.

2 The SEE trade-off optimization problem can be formulated as follows

$$
\begin{array}{ll}
\max _{p} q=\sum_{i=1}^{M} R_{i}(p) / P_{\text {tot }}(p) \\
C 1: R_{i}(p) \geq R_{i}^{\text {req }} & \forall i=1, \ldots, M, \\
C 2: 0 \leq p_{i} \leq p_{i}^{\max } & \forall i=1, \ldots, M
\end{array}
$$

where $R_{i}^{\text {req }}$ represents the minimum rate required by user $i$ and the unit of $q$ is bits/Joule/Hz.

MST is a Mixed Integer Nonlinear Programming (MINLP) problem in which the CSI transmitted be- tween the transmitter and the eavesdropper is exponentially distributed. The path tracking algorithm is proposed to solve MST problem [16]. The difference between two convex functions is used to represent confidentiality rate $\Phi_{i}(p)$.In each iteration, information throughput $f_{i}(p)$ and eavesdropping throughput $g_{i}(p)$ are respectively determined by the lower limit $R_{i}^{(t)}(p)$ and upper $\operatorname{limit} \Gamma_{i}^{(t)}(p)$ approximate. The path tracking algorithm initialize a feasible solution $p^{(0)}$, and solve the following convex optimization problem in $t$ iterations.

$$
\begin{aligned}
& \max _{p} \Phi^{(t)}(p)=\min _{i=1, \ldots, M} \Phi_{i}^{(t)}(p) \\
& \text { s.t. } 0 \leq p_{i} \leq p_{i}^{\max } \quad \forall i=1, \ldots, M
\end{aligned},
$$

The condition for stopping the iteration is $\left|\left(\Phi^{(t)}-\Phi^{(t-1)}\right) / \Phi^{(t)}\right| \leq \varepsilon$, where $\varepsilon$ is a set parameter.

For the SEE trade-off optimization problem, bisection method and successive approximation method are used to solve the problem. The $q$ value is improved by the following:

$$
\max _{p} \sum_{i=1}^{M} R_{i}(p)-q P_{t o t}(p) .
$$

GAP [32] can solve the above problem by solving a standard convex optimization problem successively in each iteration. The condition for stopping the iteration is $\left|R_{i}(t)-q_{t o t}{ }^{(t)}\right| \leq \varepsilon_{1}$, where $\varepsilon_{1}$ is a set parameter. Solving MST is an unconditionally constrained continuous problem, similar to the process of a whale searching for an optimal. Based on WOA, penalty method is used to deal with the minimum rate requirement constraint $C 1$ in problem (7). User can be equivalent to a humpback whale, and transmission power $P$ can be equivalent to the location of search whale $X(t)$. Transmit power $P(t)$ is updated by shrinking encirclement, spiral up-dating position, and exploring prey mechanism. The fitness function for evaluating best search can be defined as

$$
\operatorname{Fitness}(p)=-\frac{\sum_{i=1}^{M} R_{i}(p)}{P_{\text {tot }}(p)}+\mu \sum_{i=1}^{M} F_{i}\left(f_{i}(p)\right) f_{i}^{2}(p),(10)
$$

where $\mu=10^{14}, f_{i}^{(p)}=R_{i}^{\text {req }}-R_{i}^{(p)}$. 


\section{Whale Optimization Algorithm}

WOA is a new population based bionic intelligent algorithm that establishes a mathematical search model based on simulated humpback whales' bubble net hunting behavior. WOA abstracts three procedures: including encircling prey, random searching, and spiral position updating.

\subsection{Surrounding Prey}

First, humpback whales need to locate their prey, but target prey's position in three-dimensional space cannot be predicted in advance. In WOA, it is assumed that the current optimal whale position is target prey. Humpback whale will update its position to current optimal during the iteration process. This behavior is formulated as a mathematical model.

$$
D=\left|C \cdot X^{*}(t)-X(t)\right|,
$$

$$
X(t+1)=X^{*}(t)-A \cdot D,
$$

where $D$ is the distance vector between current optimal and individual whale, and $X^{*}(t)$ is the current optimal position vector, $X(t)$ is the position vector of current individual whale, $t$ is the number of iterations, $A$ and $C$ are coefficient vectors. the formula is

\begin{tabular}{l}
$A=2 a \cdot r-a$, \\
\hline$C=2 \cdot r$,
\end{tabular}

where $a$ is called the convergence factor, which linearly decreases from 2 to 0 as the number of iterations $t$ increases, Update with $a=2\left(1-t / T_{\max }\right) . r$ is a uniformly distributed random vector, $r \in[0,1]$, and $T_{\max }$ is the maximum number of iterations.

\subsection{Bubble Nets Prey on Prey}

At this stage, bubble nets predation includes shrinking and spiral position renewal. The shrinking is achieved by reducing the value of in formula 13 . Mathematical model of the spiral position update is described as

$$
X(t+1)=D^{\prime} \cdot \mathrm{e}^{\mathrm{bl}} \cdot \cos (2 \pi l)+X^{*}(t),
$$

where $D^{\prime}=\left|X^{*}(t)-X(t)\right|$ is the distance vector be- tween individual whale and current optimal, $b$ is a constant, and $l \in[-1,1]$.

Whale shrink encircling circle while moving along the spiral path. In order to simulate this synchronization process, WOA select the same probability $p$ for contraction and spiral position update. Mathematical model is expressed as follows

$$
X(t+1)=\left\{\begin{array}{cc}
X^{*}(t)-A \cdot D & p<0.5 \\
D \cdot e^{b l} \cdot \cos (2 \pi l)+X^{*}(t) & p \geq 0.5
\end{array}\right.
$$

\subsection{Exploring New Prey}

When $|A|>1$,it indicates that whale has deviated from the shrinking, which whale needs to randomly select the position of a whale to replace the target prey to update its position. Humpback whale is forced to leave the target prey, by which WOA can expand the search space and realize global search. Mathematical model is as follows

$$
\begin{aligned}
& D=\left|C \cdot X_{\text {rand }}-X(t)\right|, \\
& X(t+1)=X_{\text {rand }}-A \cdot D,
\end{aligned}
$$

where $X_{\text {rand }}$ is the randomly selected position vector of individual whale.

\section{Improvement}

WOA only provides each parameter's basic setting and fundamental strategy choice, and the original algorithm is not enough to solve MINLP problems. Therefore, we propose Cosine Compound Whale Optimization Algorithm (CCWOA).

\subsection{Nonlinear Convergence Factor and Inertia Weight}

WOA is a metaheuristic algorithm based on population iteration, its advantage is that it can converges quickly in the iterative process. The development and exploration ability of WOA largely depends on the change of convergence factor $a$. The convergence speed is slow and it is unable to calculate high complexity when $a$ decreases linearly in the iteration. Analysis displays that a larger convergence factor is more capable of global search and a smaller conver- 
gence factor is more suit-able for local development. In the evolutionary search process of WOA, convergence factor $a$ decreases linearly with the number of iterations, which can not fully reflect the actual optimization search process [17]. Because cosine function is nonlinear in the period interval [8], we consider that factor $a$ decreases to a small value with iteration in the early stage of search, and increases slowly in the later stage. Factor $a$ is defined as

$$
a=\left(a_{\max }-a_{\min }\right) \cdot \cos \left(t / T_{\max }\right)+2\left(1-t / T_{\max }\right),
$$

where $a_{\max }$ and $a_{\min }$ are the variables of the control the parameters. The difference in the selection parameters of $a_{\max }$ and $a_{\min }$ will also affect convergence factor. The selection of parameters will be specified in the experimental part, $t$ is the current iteration number, $T_{\max }$ is the maximum iteration number. The new convergence factor $a$ can slow down the convergence efficiency at the beginning, and then increase nonlinearly. Experiments show that it has better convergence characteristics than other periodic functions when applied to the convergence factor. $t / T_{\max }$ in original WOA can be defined as convergence efficiency.

From formulas (12) and (16), it can be known that the original WOA does not consider the attractiveness of prey to guide different whales for position updates during the iteration process. The new whale position is only determined by the current target whale position and the global optimal whale position, which easily leads to algorithm falling into the local optimum. Inspired by PSO, we use the inertia weight idea in PSO to reconstruct local optimum position. At the beginning of the iteration, optimal position is adjusted with a larger weight coefficient to expand search field. At the later of the iteration, a smaller weight coefficient is used to fine tune the optimal position to achieve rapid convergence. The specific improvement formula is as follows.

$$
\begin{aligned}
& n(t)=\left(a_{\max }-a_{\min }\right) \cdot \cos \left(t / T_{\max }\right), \\
& X(t)=n \cdot X^{*}(t)-A \cdot D \quad p<0.5|A| \leq 1, \\
& X(t)=n \cdot X_{\text {rand }}-A \cdot D \quad p<0.5|A|>1,
\end{aligned}
$$

where $a_{\max }$ and $a_{\min }$ are variables, $n$ is the weight coefficient.

\subsection{Improved Spiral Update Position Model}

In the original WOA, spiral update position in formula (15) uses a logarithmic spiral update method. In [27], Archimedes spiral is used instead of logarithmic spiral, and we refer to this spiral updating method. When spiral updates position, step distance may exceed search range, logarithmic spiral is to gather the population together quickly. In this process, best whale's position may be lost due to the iteration step too large. Archimedes spiral is a trajectory generated by a point, leaving a fixed point at a constant speed while rotating around the fixed point at a fixed angular velocity [27]. After the spiral pitch exceeds range, search space can still be traversed uniformly, reducing the possibility of losing best whale position. Formula [38] is further improved as.

$$
X(t+1)=D^{`} \cdot b l \cdot \cos (2 \pi l)+X^{*}(t) .
$$

By analyzing the formula (23), we can find local optimum in the current space by uniformly traversing search space. It is difficult to guarantee that it is the global optimum in the whole space. For this problem, the algorithm design adds sine $X(t)$.The value of sine belongs to interval $[-1,1]$, which is equivalent to adding random value. The randomness of whale $X(t)$ position is enlarged and the probability of jumping out of local optimal solution is improved. Therefore, adding sine $X(t)$ to the formula (23), sine global search can assist the local development of cosine, greatly reducing the blind spots in optimization and avoiding the loss of optimal. The local development of cosine will make up for the slow convergence speed of the global search of sine, which effectively improves algorithm's efficiency. The specific formula for improvement is as follows.

$$
X(t+1)=D^{\prime} \cdot b l \cdot \cos (2 \pi l)+\sin X(t)+X^{*}(t) .
$$

\section{Algorithm 1. CCWOA's pseudo code}

Initialize the whale population $t=1, \mathrm{i}=1, \ldots, \mathrm{N}$, the number of iterations $t=1$, the maximum number of iterations $T_{\max }$.

Calculate the search whale's fitness and deter-mine the best search whale $X^{*}(t)$. 
for $k=1$ to $\mathrm{M}$ do

Update the convergence factor $a$, the constant variables $A$ and $C$, the helical constant $l$ and the probability $p$.

if $p<0.5$ then

if $|A|<1$ then

Update $D$ according to formulas 11,17 and update in 18.

else

Update $D$ and $X_{\text {rand }}$ according to 23 .

Update $X$ according to 21 .

end if

else

Update the current whale position, $X$ and $D$ according to formulas 22 and 24 .

end if

end for

Calculate the fitness of each whale and update the best whale position.

Carried out at $t=t+1$.

Until $t>T_{\max }$, determine whether the termination conditions are met.

Output optimal.

Based on WOA, CCWOA introduces a new convergence factor to speed up the surrounding prey's speed, which adds inertial weight to optimize the new whale position. In the process of narrowing the en-circling circle of the spiral, the archiedean spiral introduced can uniformly find the better solution in the current environment. The random value of the sine will increase the probability of jumping out of the local optimum. The CCWOA has been dramatically improved in accelerating convergence's accuracy and jumping out of the local optimum. According to the above description, the pseudocode of the CCWOA is shown in algorithm 1 below.

\subsection{Analysis of the Complexity of the Improved Algorithm}

In WOA, the computational complexity of calculating fitting function is $O(N \cdot \mathrm{Dim}) . N$ is whale populations and Dim is the dimension of search agent [37]. In each iteration, updating the position vectors of all whales requires $O(N \cdot \mathrm{Dim})$ complexity, so the com- putational complexity of WOA is $O\left(N \cdot \mathrm{Dim} \cdot T_{\text {max }}\right)$. Among them, $T_{\max }$ is the maximum number of iterations. According to the pseudo code of CCWOA above, newly added nonlinear convergence factor and inertia weight mainly increase the calculation amount of $O\left(N \cdot \operatorname{Dim} \cdot T_{\max }\right) \cdot$ Although the newly added sinusoidal whale position increases the probability of jumping out of the local optimum, it also increases the amount of calculation of $O\left(N \cdot T_{\max }\right)$.The computational complexity of CCWOA is $O\left(N \cdot \mathrm{Dim} \cdot T_{\max }+N \cdot T_{\max }\right)$. If we encounter high-dimensional problems in real network communication, such as the interference environment considered in this paper, the calculation amount of $O\left(N \cdot T_{\max }\right)$ can be ignored, so the calculation complexity of CCWOA and WOA are approximately the same.

\section{Simulation Experiment and Result Analysis}

In order to verify the power allocation optimization capability of CCWOA in IWN, we consider to test from the following three aspects.

1 This paper tests the computational power of CCWOA by adopting 8 classical benchmark functions in Table 1. Benchmark functions can be divided into two groups: unimodal, multimodal functions. Functions $f_{1}(x)$ to $f_{4}(x)$ are typical unimodal because they have only one global optimum. These functions allow to evaluate the exploitation capability of the investigated metaheuristic algorithms. The functions $f_{5}(x)$ to $f_{8}(x)$ are multimodal functions. Unlike unimodal functions, multimodal functions include many local optimum whose number increases exponentially with the problem size. By comparing the optimization capabilities of CCWOA, WOA, GWO and MPA in 8 functions, the improved algorithm's advantages and disadvantages are explained.

2 We compare this algorithm with other power allocation algorithms in interference network, and research the optimization performance of the algorithm as a whole. First, the same initial parameters are set to ensure the scientific performance of the experiment. The initial population of whales is $N$, the maximum iteration number is $T_{\max }$, the iteration number is $t$. It is compared with the GWO, 
Table 1

Testing functions

\begin{tabular}{|c|c|c|c|}
\hline Function & Function Expression & Range & $F_{\min }$ \\
\hline F1 & $f_{1}(x)=\sum_{i=1}^{d} x_{i}^{2}$ & {$[-100,100]$} & 0 \\
\hline $\mathrm{F} 2$ & $f_{2}(x)=\sum_{i=1}^{d}\left(\sum_{j-1}^{j} x_{j}\right)^{2}$ & {$[-100,100]$} & 0 \\
\hline F3 & $f_{3}(x)=\max _{i}\left\{\left|x_{i}\right|, 1 \leq i \leq n\right\}$ & {$[-100,100]$} & 0 \\
\hline F4 & $f_{4}(x)=\sum_{i=1}^{d-1}\left(100\left(x_{i+1}-x_{i}^{2}\right)+\left(x_{i}-1\right)^{2}\right)$ & {$[-30,30]$} & 0 \\
\hline F5 & $f_{5}(x)=\frac{1}{4000}\left(\sum_{i=1}^{n}\left(x_{i}^{2}\right)\right)-\left(\prod_{i=1}^{n} \cos \left(\frac{x_{i}}{\sqrt{i}}\right)\right)+1$ & {$[-600,600]$} & 0 \\
\hline $\mathrm{F} 6$ & $\begin{array}{l}f_{6}(x)=\frac{\pi}{n}\left\{10 \sin \left(\pi y_{1}\right)+\sum_{i=1}^{n=1}\left(y_{i}-1\right)^{2}\left[1+10 \sin ^{2}\left(\pi y_{i+1}\right)\right]+\left(y_{n}+1\right)^{2}\right\} \\
+\sum_{i=1}^{n} \mu\left(x_{i}, 10,100,4\right) \\
y_{i}=1+\frac{x_{i}+1}{4} ; \mu\left(x_{i}, a, k, m\right)=\left\{\begin{array}{l}k\left(x_{i}-a\right)^{m}, x_{i}>a \\
0,-a<x_{i}<a \\
k\left(-x_{i}-a\right)^{m}, x_{i}<-a\end{array}\right.\end{array}$ & {$[-50,50]$} & 0 \\
\hline Fr & $\begin{array}{l}f_{7}(x)=0.1\left\{\sin ^{2}\left(3 \pi \mathrm{x}_{1}\right)+\sum_{i=1}^{n}\left(x_{i}-1\right)^{2}\left[1+\sin ^{2}\left(3 \pi x_{i}+1\right)\right]+\left(\mathrm{x}_{\mathrm{n}}-1\right)^{2}\left[1+\sin ^{2}\left(2 \pi \mathrm{x}_{\mathrm{n}}\right)\right]\right\} \\
+\sum_{i=1}^{n} u\left(x_{i}, 5,100,4\right)\end{array}$ & {$[-50,50]$} & 0 \\
\hline F8 & $f_{8}(x)=-\sum_{i=1}^{5}\left[\left(X-a_{i}\right)\left(X-a_{i}\right)^{T}+c_{i}\right]^{-1}$ & {$[0,10]$} & -10.15 \\
\hline
\end{tabular}

WOA, PFP and MPA on the MST optimization. Meanwhile, it compares with the GAP, WOA and CCWOA on the balance of SEE.

3 We test the different improved sub strategies of the algorithm, and analyze the influence of different sub strategies on the final optimization performance. For the variables $a_{\max }$ and $a_{\min }$ used in $a$ and inertia weight, we design to choose different parameter values. Different values will have a significant impact on the rate of convergence, so finding the appropriate parameter value is one of the key points of our study.

\subsection{Comparison of Benchmark Functions}

We analyzed the results of CCWOA, WOA, GWO, and MPA after 30 independent experiment runs. Experiments record the average, and standard deviations for the best fitness of the benchmark function.The experimental results are shown in Table 2.

After 30 experiments, the best fitness values for CCWOA, WOA, GWO, and MPA were used to plot convergence curves and observe differences between the four algorithms, as shown in Figure 2,3. 
Table 2

Performance comparison between WOA and CCWOA

\begin{tabular}{c|c|c|c|c|c|c|c|c|c}
\multirow{2}{*}{ Function } & \multicolumn{2}{|c|}{ WOA } & \multicolumn{2}{c|}{ CCWOA } & \multicolumn{2}{c|}{ MPA } & \multicolumn{2}{c}{ GWO } \\
\cline { 2 - 12 } & AVE & STD & AVE & STD & AVE & STD & AVE & STD \\
\hline F1 & $2.378 E-75$ & $2.833 \mathrm{E}-73$ & $3.04 \mathrm{E}-149$ & $8.19 \mathrm{E}-147$ & $6.154 \mathrm{E}-24$ & $6.145 \mathrm{E}-23$ & $4.248 \mathrm{E}-28$ & $1.049 \mathrm{E}-27$ \\
\hline F2 & $1.942 \mathrm{E}-51$ & $1.847 \mathrm{E}-50$ & $9.734 \mathrm{E}-65$ & $1.114 \mathrm{E}-65$ & $3.156 \mathrm{E}-13$ & $4.449 \mathrm{E}-12$ & $1.587 \mathrm{E}-17$ & $5.368 \mathrm{E}-17$ \\
\hline F3 & 77.098 & 77.229 & $2.02 \mathrm{E}-119$ & $6.16 \mathrm{E}-117$ & $5.716 \mathrm{E}-09$ & $6.729 \mathrm{E}-08$ & $6.515 \mathrm{E}-077$ & $3.133 \mathrm{E}-06$ \\
\hline F4 & 27.788 & 27.877 & 28.435 & 28.495 & 26.049 & 25.342 & 27.149 & 27.166 \\
\hline F5 & 0.451 & 0.612 & $2.220 \mathrm{E}-16$ & $3.586 \mathrm{E}-14$ & $1.110 \mathrm{E}-18$ & $2.220 \mathrm{e}-17$ & 0.342 & 0.378 \\
\hline F6 & 0.004 & 0.005 & $9.798 \mathrm{E}-07$ & $1.362 \mathrm{E}-06$ & $7.600 \mathrm{E}-09$ & $1.487 \mathrm{E}-08$ & 0.021 & 0.032 \\
\hline F7 & 0.307 & 0.342 & $2.571 \mathrm{E}-07$ & $3.452 \mathrm{E}-06$ & 0.057 & 0.045 & 0.539 & 0.513 \\
\hline F8 & -7.139 & -7.123 & -10.153 & -10.239 & -10.153 & -10.324 & -7.983 & -8.181 \\
\hline
\end{tabular}

\section{Figure 2}

Unimodal function

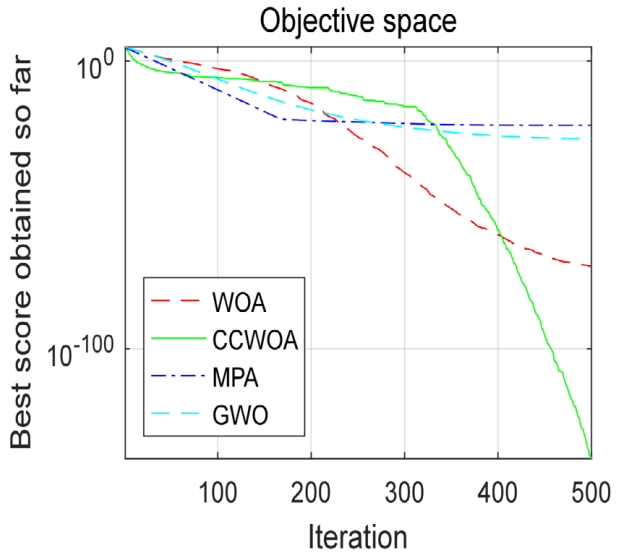

(a) F1

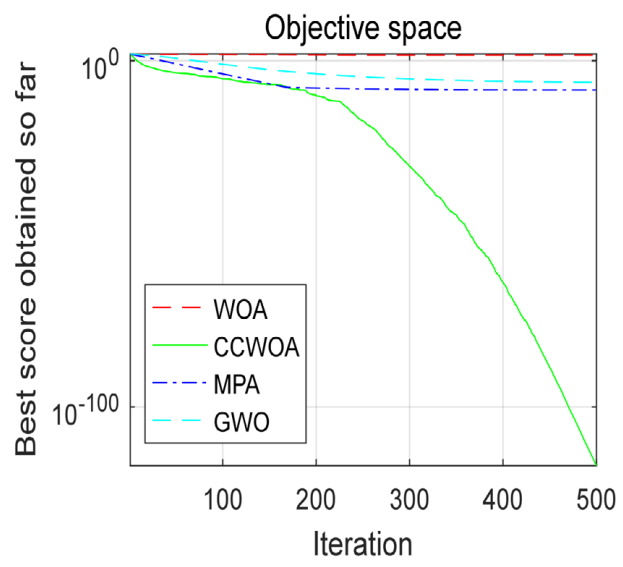

(c) F3

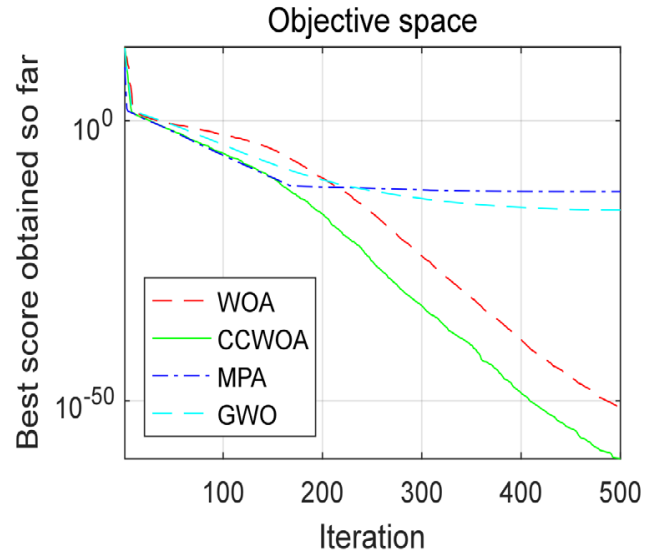

(b) F2

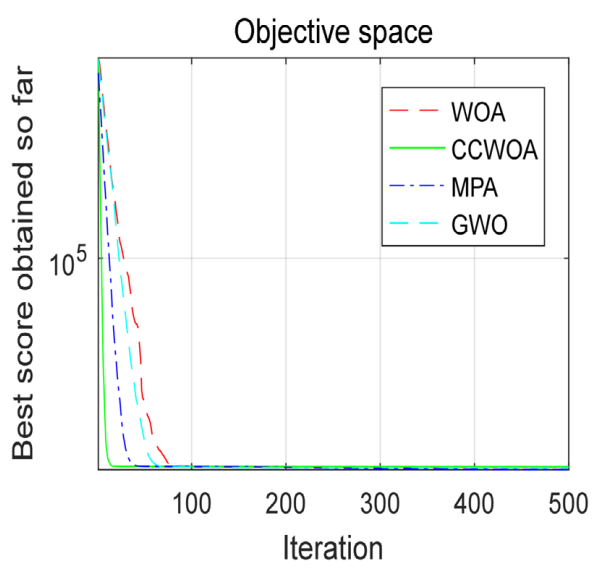

(d) F4 


\section{Figure 3}

Multimodal function

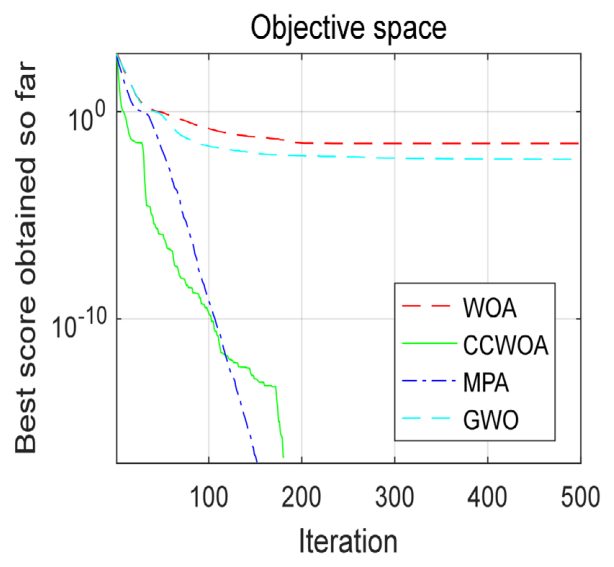

(a) F5

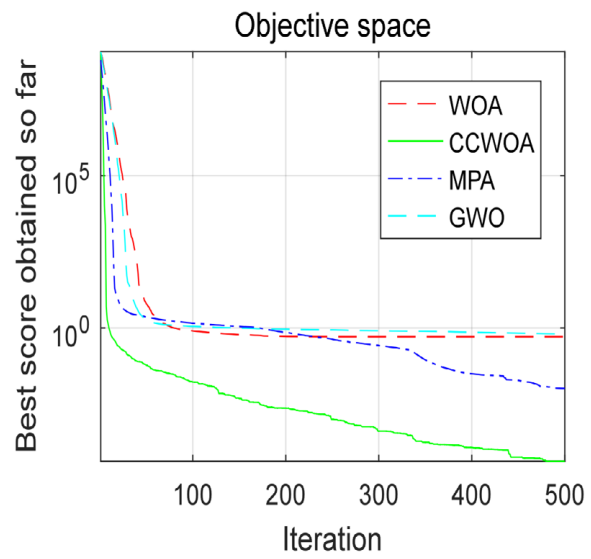

(c) Fry

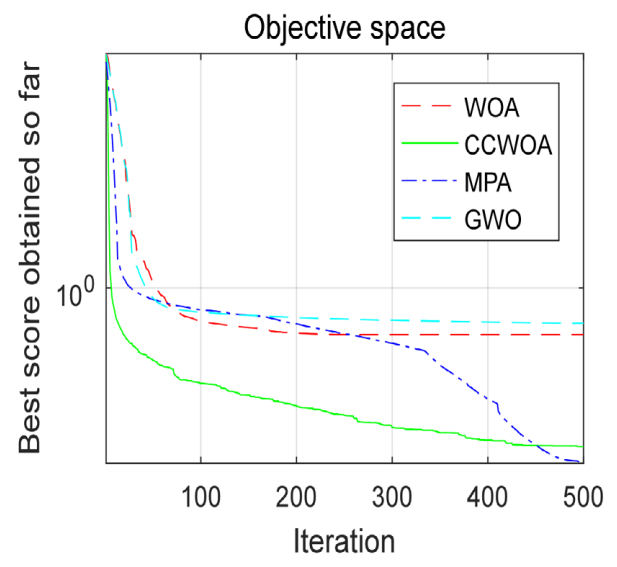

(b) F6

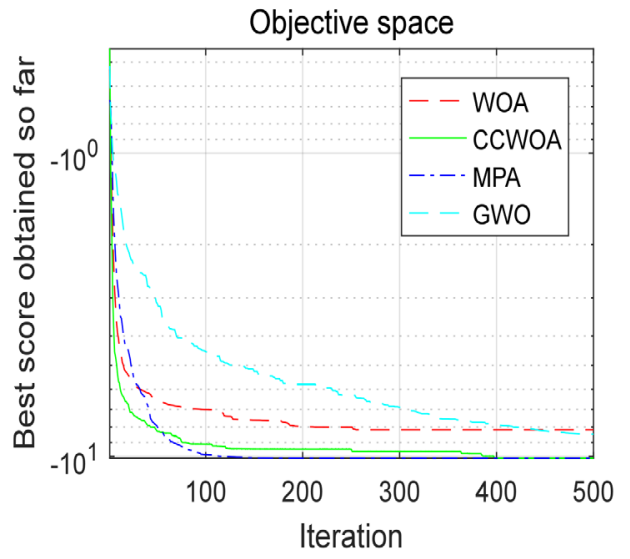

(d) F8
It can be seen from the data in table 2 and the benchmark functions in figures 2 and 3 that compared with the other three algorithms, CCWOA algorithm has good performance in optimization speed and convergence results. At the same time, it is not difficult to find that for functions $f_{1} \sim f_{4}$, GWO and MPA are better than WOA, and they are slightly worse than CCWOA. Since $f_{1} \sim f_{4}$ are unimodal functions, table 2 and figures 2 and 3 can show that CCWOA is effective in solving most single optimal problems. Among the four algorithms in $f_{7}$ function, CCWOA is the best, MPA is the second, and WOA is better than GWO.
The convergence curves of CCWOA and MPA in $f_{5}$ and $f_{6}$ functions are almost the same, and the optimization speed is fast decreasing, which shows that the optimization effect of the two algorithms is very good. The optimization effect of WOA and GWO is much lower than the above two. It is worth noting that the best score of CCWOA is slightly lower than that of MPA. However, in the $f_{8}$ function, CCWOA and MPA get the same optimal score.

On the whole, the optimization effect of CCWOA on benchmark function is significant, and CCWOA can improve the accuracy of solving the problem and the 
stability of the algorithm. At the same time, it can be seen that the optimization performance of MPA in $f_{5}$ and $f_{6}$ benchmark function is slightly better. The design and optimization principles of these two algorithms are quite different, and the ability of multi value optimization needs to be further studied.

\subsection{Performance Comparison in Power Allocation in Interference Networks}

\subsubsection{Performance Comparison in MST}

This paper uses the same optimized power allocation simulation parameters in [26], which the initial feasible point $p_{i}^{(0)}$ of the path tracking process is initialized randomly:

$$
p_{i}^{(0)}=\rho\left(p_{i}^{\max }-p_{i}^{\min }\right)+p_{i}^{\min } .
$$

Meanwhile, $p_{i}^{(0)}=0$ represents the minimum transmit power of user is $i, \rho \in[0,1]$.The initial whale population size is $N=30$, the maximum number of iterations $T_{\max }=500$, the number of runs 50 times.

Compared with GWO, WOA, PFP and MPA, it is optimized on the minimum secrecy throughput. The experimental results are shown in Figure 4.

Figure 4 is a comparison of the MST in the communication link where the eavesdropper is legally present. The higher the MST rate, the smaller the eavesdropping throughput in the link, and the larger the user's security throughput. The link communication safety factor that represents transmission information is higher. As can be seen from Figure 4, the throughput rate of CCWOA is higher than that of the original WOA. It is proved that the introduced $a$ and inertia weight are effective, which can make the algorithm have good optimization accuracy.

In Figure 4, we can also see that the MST of GWO is lower than that of WOA and CCWOA, because the gray wolf population has a strict hierarchy. Individuals can't update their targets randomly. When exploring prey, they need to report to the high level nodes every time, which reduces the overall efficiency. Meanwhile, the optimization effect of MPA is similar to GWO. It can be seen that MPA has obvious rate pause and slow down in the previous iteration, and then increases. In the early stage of MPA, Brownian motion and Lévy movement are used to explore prey to maximize the encounter with the optimal value.

\section{Figure 4}

Comparison of five algorithms for MST optimization

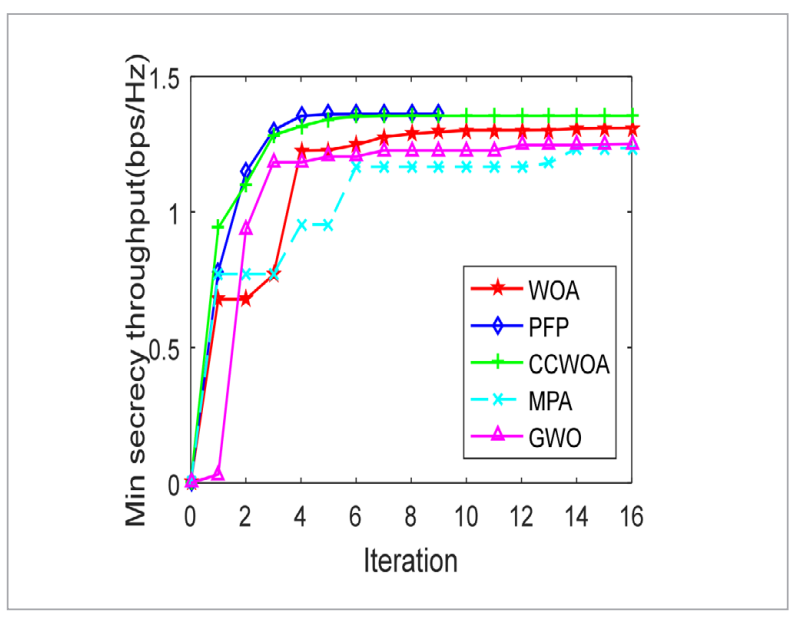

This also leads to the bad effect of MST optimized by MPA. The throughput rate of PFP is approximately the same as that of CCWOA, and is higher than that of WOA. PFP is a path tracing algorithm. In each iteration, the solution of the previous convex problem needs to be called, which increases the amount of information processing. In WOA, humpback whales update their positions synchronously according to the best whale position, which is not affected by the gradient.

In the interference network scenario considered in this paper, the computational complexity of PFP is $O\left(T_{\max } \cdot\left(N^{2} \cdot N^{2.5}+N^{3.5}\right)\right)$ [32]. Moreover, $T_{\max }=9$ and $N=30$. According to the conclusion in Section 3.3, the computational complexity of WOA and CCWOA is approximately $O\left(T_{\max } \cdot \mathrm{Dim} \cdot N\right)$ when dealing with high dimensional problems such as interference network scenes. $T_{\max }=16$ and $N=30$. It can be seen that the computational complexity of WOA and CCWOA is significantly lower than that of PFP. Furthermore, the MST of CCWOA is faster than WOA. Based on the above analysis, CCWOA has a better performance than other algorithms when optimizing the power allocation problem in interference networks.

\subsubsection{Performance Comparison in SEE}

Moreover, the SEE can also fully reflect the performance of the algorithm. The tradeoff between EE and $\mathrm{SE}$ can be expressed by maximizing SEE under the constraints of minimum rate requirement and trans- 
mission power budget. Energy efficiency is the ratio of throughput to total network power consumption. High energy efficiency means an increase in throughput, with the total power consumption of network unchanged. When the maximum iteration times $T_{\max }$ and population size $n$ are the same, CCWOA and WOA compare the advantages and disadvantages of the two algorithms in dealing with SEE problems. As shown in Figure 5.

\section{Figure 5}

The ability of CCWOA and WOA to obtain SEE

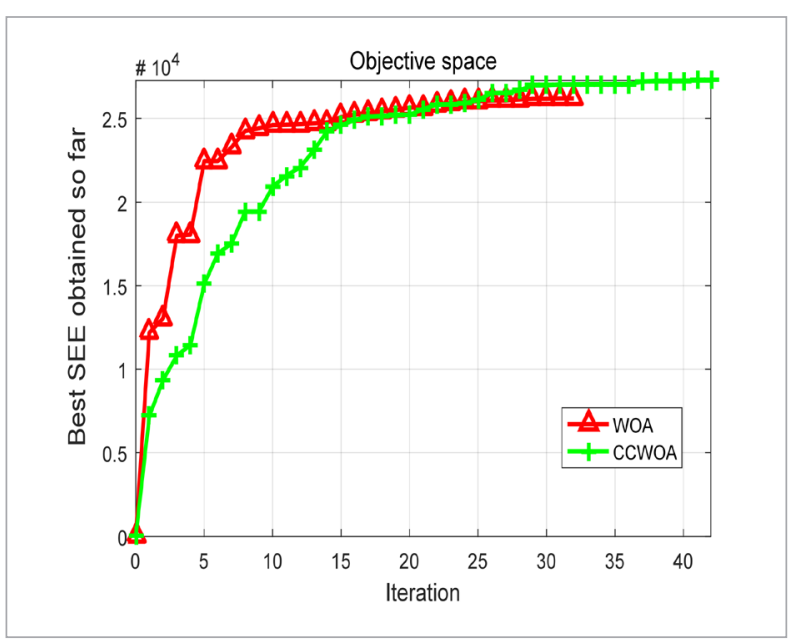

Figure 5 displays the situation of CCWOA and WOA in dealing with the security energy allocation efficiency in each iteration. Score is proportional to the optimization effect of algorithm. Looking at Figure 5, it is found that the SEE of CCWOA is about $4 \%$ higher than that of WOA, when the number of iterations stopped is approximately the same.

In addition, to fully illustrate the advantages and disadvantages of CCWOA to balance energy efficiency. This algorithm uses the same simulation parameters with GAP algorithm and WOA to compare the optimization energy efficiency. Energy efficiency is also an important reference index of link security. When the energy efficiency is higher, it means that the security throughput of the link is larger and the probability of secret information being stolen will be reduced. The algorithm is terminated when $\left|q^{(t)}-q^{(t-1)}\right| \leq 10^{-6}$ is three times in a row. As shown in Figure 6.

We plot the energy efficiency tradeoff curve in Figure 6 and observe that energy efficiency decreases as higher minimum rates are required. It can be seen
Figure 6

CCWOA optimizes the consequent of Energy Efficiency

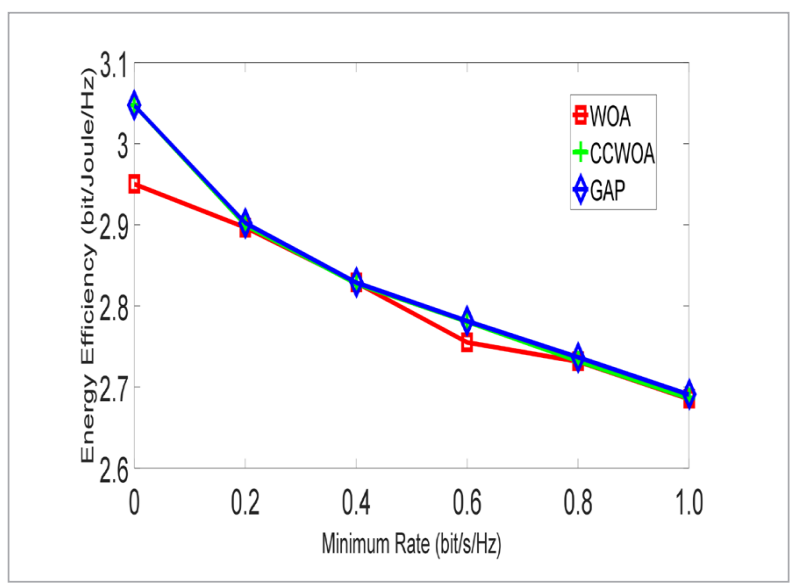

from the figure that CCWOA and GAP curves approximately overlap, in which WOA curve is lower than the two. It indicates that the optimization effect of CCWOA is similar to GAP and better than WOA. The optimized energy efficiency of the WOA is lower than the two. However, we must stress that the proposed WOA-based algorithm requires a single-loop operation, but the GAP is a two-loop scheme, where outer loop is to update the energy efficiency and inner loop is to optimize transmit power. Moreover, each inner loop needs to find the solution to a sequence of approximate convex problems, which increases computational complexity. CCWOA is a non-gradient method. Compared with GAP algorithm, CCWOA has more advantages in optimizing energy efficiency index, when the actual network has less calculation.

This conclusion can also be seen by comparing the computational complexity of three methods. After complement the above analysis, the computational complexity of CCWOA is $O\left(T_{\max } \cdot N \cdot(2 M)\right)$. In the same situation, the computational complexity of GAP is $\left.O\left(T_{1} T_{2}\left(M^{2}(2 M)^{2.5}\right)+(2 M)^{2.5}\right)\right)$, the number of iterations of outer loop of GAP $T_{1}=14$, which the number of iterations of inner loop $T_{1}=12$. Comparing with GAP, CCWOA has a very low computational complexity, which is crucial for practical applications. Thus, CCWOA has better competitiveness.

\subsection{The Improvement Strategy of the CCWOA}

In order to analyze the influence of different sub strategies of CCWOA on the performance of the al- 
gorithm. The algorithm is divided into four sub-strategies $C 1-C 4$, by which the degree of data rate optimization of different sub-strategies is analyzed. In the experiment, $C 1$ is set to only change the convergence factor $a, C 2$ is set to introduce only the inertial weight $n, C 3$ is set to replace the logarithmic spiral with Archimedes spiral and the sine vector is added, and $C 4$ is set to the improved full CCWOA. This section sets the same parameters as above, compares the impact of $C 1-C 4$ on power allocation. The result is shown in Figure $\%$

\section{Figure 7}

The degree of impact of different sub-strategies on performance in CCWOA

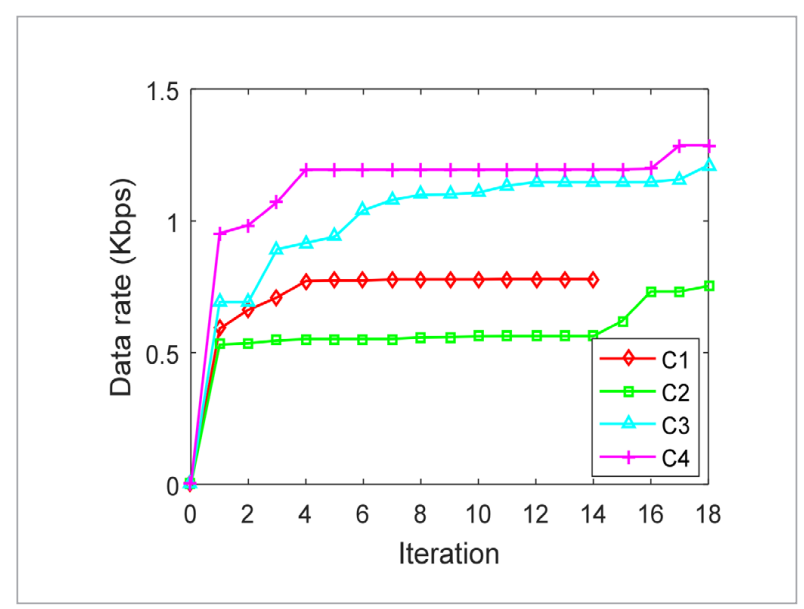

Figure 7 describes the impact of different sub-strategies on the data rate. It can be seen that $C 4$ has the best performance for optimizing the data rate, and $C 2$ has less impact on the algorithm performance. The $C 1$ and $C 3$ have a general increase in the optimized data rate, which indicates that the nonlinear convergence factor, spiral model and sine vector have a significant impact on the optimization of algorithm. Under the same parameters and environment, the overall CCWOA has faster convergence speed, which displays the rationality and effectiveness of algorithm combined with three improved methods.

When designing the nonlinear convergence factor $a$, we set $a_{\max }$ and $a_{\text {min }}$ as variables, and record $\left(a_{\max }-a_{\min }\right)$ as $r . r$ is mainly to balance the role of global exploration and local development. It has a significant impact on the convergence rate of the overall iteration when $r$ is selected with a different value. Ac- cording to the [16], when $r \in[0,1.4]$, the search position will not move out of dimensional range, and $r$ can play a normal regulatory role in this interval. This section divides the interval $[0,1.4]$ into four sub-intervals, as shown in Table 3. The specific experimental results are shown in Figure 8.

Table 3

Interval division

\begin{tabular}{c|c}
\hline Condition name & Subinterval \\
\hline Case-a & {$[0,0.3]$} \\
\hline Case-b & {$[0.4,0.6]$} \\
\hline Case-c & {$[0.7,1.0]$} \\
\hline Case-d & {$[1.1,1.4]$} \\
\hline
\end{tabular}

Figure 8

The effect of different interval $r$ on $a$ and inertia weight

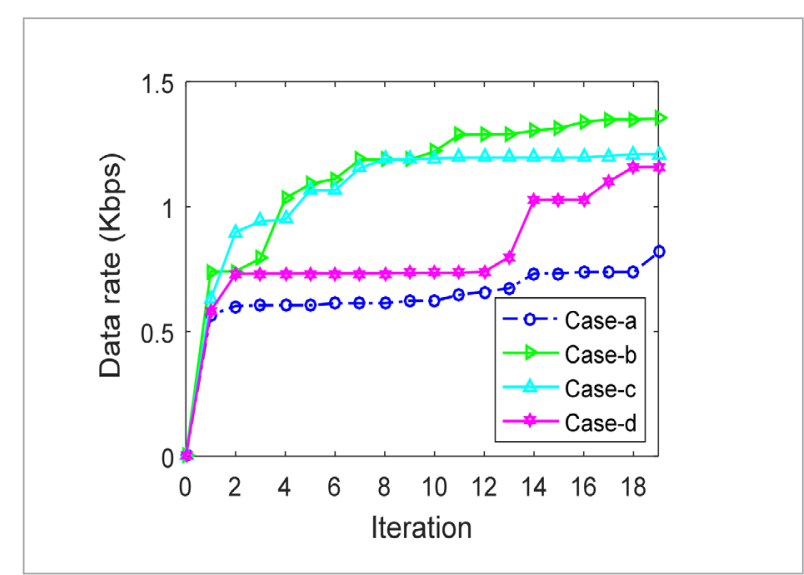

The influence of $r$ value on the data rate in different intervals is shown in Figure 8. $r$ improves the data rate best when it is in the $b$ interval. When $r$ is in the interval of segment $a$, the value is too small, and nonlinear convergence factor and inertia weight are not changed much. The performance of the convergence factor is closer to global exploration when $r$ is in the $c-d$ interval. It has been developing new areas, which is obviously not suitable for use in this scenario. The improvement effect of this algorithm is most suitable when $r \in[0.4,0.6]$. After many random tests, it is found that the probability of normal distribution is the largest when $r$ is at 0.5. Thus, the value of $\left(a_{\max }-a_{\min }\right)$ in formula 23 is determined to be 0.5 . 


\section{Conclusion and Future Work}

Aiming at the communication security problem in the interference network, this paper uses an improved WOA to optimize the power allocation of the network. The efficiency of channel-safe signal transmission is effectively enhanced. We draw on the experience of strengthening the swarm intelligence algorithm and analyze the WOA search mechanism. Meanwhile, a CCWOA that uses cosine vector nonlinear convergence factor, increases inertia weight, and improves spiral search path is proposed. It is found that the convergence performance of CCWOA is better than that of WOA by testing on 8 benchmark functions. Compared with existing algorithms, CCWOA has a obvious optimization effect on performance indicators, such as MST and SEE. Then analyze the sub-strategies in turn and compare the impact of different improvements on the algorithm. It is concluded that the improvement of algorithm not only makes the searching of agents more purposeful, but also prevents the algorithm from falling into local optimum. This indicates the rationality and effectiveness of CCWOA in power allocation.

\section{References}

1. Abdelbasset, M., Manogaran, G., Elshahat, D., Mirjalili, S. M. Integrating the Whale Algorithm with Tabu Search for Quadratic Assignment Problem: A New Approach for Locating Hospital Departments. Applied Soft Computing, 2018, 73, 530-546. https://doi. org/10.1016/j.asoc.2018.08.047

2. Aljarah, I., Faris, H., Mirjalili, S. Optimizing Connection Weights in Neural Networks Using the Whale Optimization Algorithm. Soft Computing, 2018, 22, 1-15. https://doi.org/10.1007/s00500-016-2442-1

3. Bassily, R., Ekrem, E., He, X., Tekin, E., Xie, J., Bloch, M. R., Ulukus, S., Yener, A. Cooperative Security at the Physical Layer: A Summary of Recent Advances. IEEE Signal Processing Magazine, 2013, 30, 16-28. https:// doi.org/10.1109/MSP.2013.2260875

4. Bozorgi, S. M., Yazdani, S. IWOA: An Improved Whale Optimization Algorithm for Optimization Problems. Journal of Computational Design and Engineering, 2019, 6. https://doi.org/10.1016/j.jcde.2019.02.002

5. Chen, H., Xu, Y., Wang, M., Zhao, X. A Balanced Whale Optimization Algorithm for Constrained Engineering De-
The cosine nonlinear convergence factor of CCWOA is effective in global multi-objective optimization. However, there are more possibilities for the selection of the convergence factor. In the future work, we will try to use properly the sine cosine mixed nonlinear convergence factor reasonably without changing the original effect of the convergence factor. The spiral method used in the development stage also has the possibility of further expansion. From the design level of the algorithm, it can also include mutation and other evolutionary operations. In addition, in the future work, we plan to build a memetic algorithm framework, which integrates Q-learning with CCWOA. The purpose is to store the location of each prey in the $\mathrm{Q}$ table. With the increase of learning times, the location of prey will be more accurate. For swarm intelligence optimization algorithm group, we hope the emergence of CCWOA algorithm can provide some ideas and directions for future research.

\section{Acknowledgement}

This work was partially supported by the National Natural Science Foundation of China (11461038), the Innovation Foundation of Colleges and Universities in Gansu Province (2020A-033).

sign Problems. Applied Mathematical Modelling 2019, 71, 45-59. https://doi.org/10.1016/j.apm.2019.02.004

6. Chorti, A., Perlaza, S. M., Zhu, H., Poor, V. H. On the Resilience of Wireless Multiuser Networks to Passive and Active Eavesdroppers. IEEE Journal on Selected Areas in Communications, 2013, 31, 1850-1863. https://doi. org/10.1109/JSAC.2013.130917

7. Eappen, G., Shankar, T. Multi-Objective Modified Grey Wolf Optimization Algorithm for Efficient Spectrum Sensing in the Cognitive Radio Network. Arabian Journal for Science and Engineering, 2020. https://doi. org/10.1007/s13369-020-05084-3

8. Eberhart, R. C., Shi, Y. Comparing Inertia Weights and Constriction Factors in Particle Swarm Optimization, 2000, 1, 84-88.

9. Ekrem, E., Ulukus, S. Secrecy in Cooperative Relay Broadcast Channels. IEEE Transactions on Information Theory, 2011, 57, 137-155. https://doi.org/10.1109/ TIT.2010.2090215

10. Elaziz, M. A., Oliva, D. Parameter Estimation of Solar Cells Diode Models by an Improved Opposition-Based 
Whale Optimization Algorithm. Energy Conversion and Management 2018, 171, 1843-1859. https://doi. org/10.1016/j.enconman.2018.05.062

11. Faramarzi, A., Heidarinejad, M., Mirjalili, S., Gandomi, A. H. Marine Predators Algorithm: A Nature-Inspired Metaheuristic. Expert Systems with Applications, 2020, 113377. https://doi.org/10.1016/j.eswa.2020.113377

12. Farhat, J., Brante, G., Souza, R. D., Rebelatto, J. L. Secure Energy Efficiency of Selective Decode and Forward with Distributed Power Allocation, 2015, 701-705. https://doi.org/10.1109/ISWCS.2015.7454439

13. Fragkiadakis, A., Tragos, E., Askoxylakis, I.G. A Survey on Security Threats and Detection Techniques in Cognitive Radio Networks. IEEE Communications Surveys and Tutorials 2013, 15, 428-445. https://doi. org/10.1109/SURV.2011.122211.00162

14. Gharehchopogh, F. S., Gholizadeh, H. A Comprehensive Survey: Whale Optimization Algorithm and Its Applications. Swarm and Evolutionary Computation, 2019, 48, 1-24. https://doi.org/10.1016/j.swevo.2019.03.004

15. Jameel, F., Wyne, S., Kaddoum, G., Duong, T. Q. A Comprehensive Survey on Cooperative Relaying and Jamming Strategies for Physical Layer Security. IEEE Communications Surveys Tutorials 2019, 21, 2734-2771. https://doi.org/10.1109/COMST.2018.2865607

16. Jiang, Y., Hu, T., Huang, C., Xianing, W. A Modified Particle Swarm Optimization Algorithm. 2006 International Conference on Computational Intelligence and Security, 2006, 1, 421-424. doi:10.1109/ICCIAS.-2006.294167.

17. Li, Y., Sheng, M., Yang, C., Wang, X. Energy Efficiency and Spectral Efficiency Tradeoff in Interference-Limited Wireless Networks. IEEE Communications Letters, 2013, 17, 1924-1927. https://doi.org/10.1109/ LCOMM.2013.082613.131286

18. Liang, Y., Poor, H. V., Shamai, S. Secure Communication Over Fading Channels. IEEE Transactions on Information Theory, 2008, 54, 2470-2492. https://doi. org/10.1109/TIT.2008.921678

19. Mirjalili, S., Lewis, A. The Whale Optimization Algorithm. Advances in Engineering Software 2016, 95, 5167. https://doi.org/10.1016/j.advengsoft.2016.01.008

20. Mirjalili, S., Mirjalili, S. M., Lewis, A. Grey Wolf Optimizer. Advances in Engineering Software, 2014, 46-61. https://doi.org/10.1016/j.advengsoft.2013.12.007

21. Mukherjee, A., Fakoorian, S. A. A., Huang, J., Swindlehurst, A. L. Principles of Physical Layer Security in Multiuser Wireless Networks: A Survey. IEEE Communications Surveys and Tutorials, 2014, 16, 1550-1573. https://doi.org/10.1109/SURV.2014.012314.00178
22. Nghia, N. T., Tuan, H. D., Duong, T. Q., Poor, H. V. MIMO Beamforming for Secure and Energy-Efficient Wireless Communication. IEEE Signal Processing Letters, 2017, 24, 236-239. https://doi.org/10.1109/ LSP.2017.2647982

23. Połap., D., Woźniak, M. Red Fox Optimization Algorithm. Expert Systems with Applications, 2020, $11410 \%$. https://doi.org/10.1016/j.eswa.2020.114107

24. Qiu, H., Kubo, A., Li, Z., Yue, Y. Optimal Archimedes' Spiral Interpolation for Cutter Path Generation in NC Machining of Noncircular Contours. The International Journal of Advanced Manufacturing Technology, 2008, 36, 69-82. https://doi.org/10.1007/s00170-0060809-7

25. Shannon, C. E. Communication Theory of Secrecy Systems. Bell System Technical Journal, 1949, 28, 656-'715. https://doi.org/10.1002/j.1538-7305.1949.tb00928.x

26. Sheng, Z., Tuan, H. D., Nasir, A. A., Duong, T. Q., Poor, H. V. Power Allocation for Energy Efficiency and Secrecy of Wireless Interference Networks. IEEE Transactions on Wireless Communications 2017, 1-1.

27. Shi, Y., Eberhart, R. C. A Modified Particle Swarm Optimizer, 1998, 69-73.

28. Tao, H., Gengxin, Z. Peakto-Average Power Ratio Reduction Algorithm of Artificial-Noise-Aided Secure Signal. Journal of Electronics \& Information Technology, 2018.

29. Wang, D., Bai, B., Chen, W., Han, Z. Achieving High Energy Efficiency and Physical-Layer Security in AF Relaying. IEEE Transactions on Wireless Communications, 2016, 15, 740-752. https://doi.org/10.1109/ TWC.2015.2477510

30. Wang, D., Bai, B., Chen, W., Han, Z. Energy Efficient Secure Communication Over Decodeand-Forward Relay Channels. IEEE Transactions on Communications 2015, 63, 892-905. https://doi.org/10.1109/ TCOMM.2014.2387342

31. Wang, H., Yin, Q., Xia, X. Distributed Beamforming for Physical-Layer Security of Two-Way Relay Networks. IEEE Transactions on Signal Processing, 2012, 60, 3532-3545. https://doi.org/10.1109/TSP.2012.2191543

32. Wu, X., Zhang, S., Xiao, W., Yin, Y. The Exploration/Exploitation Tradeoff in Whale Optimization Algorithm. IEEE Access 2019, 1-1. https://doi.org/10.1109/ACCESS.2019.2938857

33. Wyner, A.D. The wiretap channel. Bell System Technical Journal 1975, 54, 1355-138\%. https://doi. org/10.1002/j.1538-7305.1975.tb02040.x 
34. Yang, N., Wang, L., Geraci, G., Elkashlan, M., Yuan, J., Renzo, M. D. Safeguarding 5G Wireless Communication Networks Using Physical Layer Security. IEEE Communications Magazine 2015, 53, 20-27. https://doi. org/10.1109/MCOM.2015.7081071

35. Yao, J., Feng, S., Zhou, X., Liu, Y. Secure Routing in Multihop Wireless Ad-Hoc Networks with Decodeand Forward Relaying. IEEE Transactions on Communications, 2016, 64, 753-764. https://doi.org/10.1109/ TCOMM.2015.2514094

36. Yousri, D., Allam, D., Eteiba, M. B. Chaotic Whale Optimizer Variants for Parameters Estimation of the Chaotic Behavior in Permanent Magnet Synchronous Motor.
Applied Soft Computing, 2019, 74, 479-503. https://doi. org/10.1016/j.asoc.2018.10.032

37. Zhao, H.-Y., Wang, J.-C., Guan, X. Ant Colony System for Energy Consumption Optimization in Mobile IoT Networks. Journal of Circuits, Systems and Computers, 2020, 09, 2050150. https://doi.org/10.1142/ S0218126620501509

38. Zhu, F., Gao, F., Yao, M., Zou, H. Joint Information and Jamming-Beamforming for Physical Layer Security with Full Duplex Base Station. IEEE Transactions on Signal Processing, 2014, 62, 6391-6401. https://doi. org/10.1109/TSP.2014.2364786 